\title{
Ordnung der Wissenschaft (OdW) www.ordnungderwissenschaft.de
}

Manfred Löwisch und Viktor Kurz"

Die 2013 gegründete, vertieljährlich erscheinende Online-Zeitschrift OdW verfolgt die Open Access Strategie in der Weise, dass jedermann kostenfrei Zugang zu den Ausgaben im Internet und auch zur pdF-Version im Zeitschriftenformat hat. Die Zabl der Abonnenten liegt bei rund 650, die Zabl der täglichen Abrufe bei rund 100. Der Zugang kann auch über juris erfolgen, mit dem eine Kooperationsvereinbarung bestebt. Träger ist ein gemeinnütziger Verein. Herausgeber, Schriftleiter und Redakteure arbeiten ebrenamtlich. Die technische Abwicklung erfolgt über die Forschungsstelle für Hochschulrecht und Hochschularbeitsrecht der Universtität Freiburg im Breisgau. Gegenstand von OdW ist das Recht von Wissenschaft, Forschung und Lehre in seiner ganzen Breite.

\section{A. Gründung und Ziel}

OdW ist 2013 gegründet worden. Anlass war die Feststellung, dass in den etablierten rechtswissenschaftlichen Zeitschriften nur ein beschränkter Raum für hochschul- und wissenschaftsrechtliche Veröffentlichungen zur Verfügung steht. Zur Füllung dieser Lücke sollten die Chancen einer Online-Zeitschrift genutzt werden.

OdW will ein Forum für das Recht von Wissenschaft, Forschung und Lehre in dessen ganzer Breite sein. Die Zeitschrift erscheint ohne feste Begrenzung des Umfangs vierteljährlich und ist kostenfrei. Der Jahrgang 2014 hat 254 Seiten, der Jahrgang 2015246 Seiten, der Jahrgang 2016252 Seiten, der Jahrgang 2017292 Seiten und der Jahrgang 2018308 Seiten im DIN-A4 Format. OdW veröffentlicht auch Beiträge in englischer und französischer Sprache.

\section{B. Herausgeber, Schriftleitung, Redaktion}

Herausgeber sind mit dem Hochschul- und Wissenschaftsrecht befaßte Hochschullehrer, Richter der Verwaltungsgerichtsbarkeit, Angehörige der Ministerialverwaltung und der Verwaltung der Kirchen, Rechtsanwälte und Leiter außeruniversitärer Forschungseinrichtungen. Bei den Herausgebern liegt die Qualitätskontrolle.

Schriftleiter sind seit der Gründung Prof. Dr. Thomas Würtenberger, Professor Dr. Dr. h.c. Manfred Löwisch und Rechtsanwältin Dr. Cornelia Feldmann, alle Freiburg.

Mitglieder der Redaktion sind mit der Forschungsstelle für Hochschulrecht und Hochschularbeitsrecht der Universität Freiburg verbundene wissenschaftliche Mit-

* Prof. Dr. Dr. h.c. Manfred Löwisch leitet die Forschungsstelle für Hochschularbeitsrechtan der Universtität Freiburg im Breisgau, Viktor Kurz ist wissenschaftlicher Mitarbeiter an der Forschungsstelle. 
arbeiter, Promovierende und Studierende. Sie wirken vielfach auch nach Examen oder Promotion weiter an der Redaktionsarbeit mit.

\section{Inhalt}

Die einzelnen Nummern von OdW sind im Regelfall in die Rubriken „Aufsätze“, „Anmerkungen zur Rechtsprechung“, „Buchvorstellungen“, „Berichte und Informationen“ und „Ausgegraben“ gegliedert. Von Fall zu Fall steht OdW aber auch für die umfassende Erörterung eines Themas in einer Nummer zur Verfügung.

Das Spektrum der Aufsätze reicht von aktuellen Problemen des Hochschulrechts im engeren Sinne, über komplexe wissenschaftsrechtliche und urheberrechtliche Fragen, europarechtliche Zusammenhänge und das Hochschularbeitsrecht bis hin zu den Grundsätzen wissenschaftlicher Ethik. So ist der sechste Jahrgang mit einem Beitrag von Richterin am Bundesverfassungsgericht Susanne Baer zu „More Than Welcome.: A Berlin Call for University Ethics“ eröffnet worden. Schwerpunkthefte waren im Jahr 2014 dem Thema Biosicherheit und im Jahr 2018 wissenschaftlicher Kooperation gewidmet.

Anmerkungen zur Rechtsprechung erörtern Entscheidungen zur Hochschulverfassung, zu den Rechtsverhältnissen von Hochschullehrern und wissenschaftlichen Mitarbeitern, sowie zu Zulassungsfragen.

Die Rubrik Buchvorstellung enthält nicht nur die Besprechung von Literatur, sondern ermöglicht Autoren auch, ihre Monographien, insbesondere Dissertationen in Kurzform der wissenschaftlichen Öffentlichkeit vorzustellen. Von dieser Möglichkeit wird rege Gebrauch gemacht. Sie füllt ersichtlich eine Lücke.

Berichte und Informationen betreffen rechtspolitische Fragen, etwa die Folgen des Brexits für die Forschung in der EU, die Exellenzinitiative oder die Open Access Strategie und die Zweitveröffentlichungspflicht, aber auch das Rechtsstudium im Ausland.

Unter Ausgegraben werden ernste und weniger ernst gemeinte Äußerungen namhafter Autoren aus früherer und heutiger Zeit zu Hochschule und Wissenschaft in Erinnerung gerufen.

Im Einzelnen können die bisherigen Veröffentlichungen in OdW dem Fünf-JahresVerzeichnis auf der Webseite von OdW entnommen werden.

\section{Open Access Strategie}

OdW verfolgt die Open Access Strategie in der Weise, dass jedermann kostenfrei Zugang zu den Ausgaben im Internet und auch zur pdf-Version im Zeitschriftenformat hat. Möglich ist ebenfalls ein Abonnement auf der Webseite, um die Ausgaben automatisch zu erhalten. Aktuell liegt die Zahl der Abonnenten bei rund über 
650 Personen. Täglich werden die Seiten von OdW ca. 100 Mal pro Tag aufgerufen.

Zwischen OdW und Juris besteht eine Kooperationsvereinbarung. Juris integriert die Inhalte von OdW in die Juris Plattform und macht sie öffentlich zugänglich. Dementsprechend führt die Eingabe von Stichworten verbunden mit der Bezeichnung „OdW“ in das Rechercheportal von Juris zu den entsprechenden Stellen in OdW. Auf ein eigenes Stichwortverzeichnis ist deshalb verzichtet werden.

Die Zeitschrift und alle in ihr enthaltenen Beiträge nehmen Urheberrechtsschutz in Anspruch. Eine Verwendung zu wissenschaftlichen Zwecken unter Angabe der Quelle (Zitat) ist ausdrücklich gestattet, auch die Verlinkung als Dokument. Für jede weitergehende Verwendung ist die Zustimmung von OdW und des ausgewiesenen Autors erforderlich. OdW gebraucht den üblichen Disclaimer.

\section{E. Träger}

Träger von OdW ist der Verein Zeitschrift Ordnung der Wissenschaft e.V. Er verfolgt die Förderung von Wissenschaft und Forschung sowie der Volks- und Berufsbildung als gemeinnützige Zwecke. Die Gemeinnützigkeit ist durch Freistellungsbescheid des Finanzamts Freiburg-Stadt vom 13. 9. 2018 anerkannt.

\section{F. Aufwand}

Neben dem üblichen Aufwand für eine Vereinsgründung bestand der Gründungsaufwand vor allem in der Erstellung des Layout und der Einweisung der ersten Redaktionsmitglieder in die fach- und sachgerechte Bearbeitung der Manuskripte für die Herstellung von online- und pdf-Version.

Herausgeber, Schriftleitung und Vereinsvorstand arbeiten ehrenamtlich obne Vergütung. Die Mitglieder der Redaktion erhalten ein geringes Zeilenhonorar für die drucktechnische Bearbeitung der Manuskripte, leisten die sonstige Redaktionsarbeit aber ebenfalls ehrenamtlich.

OdW zahlt keine Autorenhonorare. Das hat sich nicht als Hemmschuh erwiesen. Vielmehr erfreut sich OdW so eines breiten, sich ständig erweiternden Kreises älterer und jüngerer Autoren aus Wissenschaft und Praxis.

Der Sachaufwand besteht in der Nutzung zweier PC im Büro der Forschungsstelle für Hochschulrecht und Hochschularbeitsrecht der Universität Freiburg, im Betreiben der Website www.ordnungderwissenschaft.de und in der elektronischen Kommunikation über die Universität mit der Adresse odw@jura.uni-freiburg.de.

Der Gründungsaufwand ist durch finanzielle Beiträge der Wissenschaftlichen Gesellschaft in Freiburg im Breisgau und der Gesellschaft für Arbeitsrecht in der Unternehmung e.V. in München gedeckt worden. 
Der laufende Aufwand, insbesondere das Zeilenhonorar für die Mitglieder der Redaktion, wird durch Mitgliedsbeiträge (100€ im Jahr) und Spenden gedeckt. Die Nutzung der PC und die elektronische Kommunikation über die Universität sind kostenfrei. 